\title{
Quality Analysis of Periapical Radiographs Taken by Dental Assistants
}

\author{
Syed Abrar Ali, Mehmood Hussain, Muhammad Shahzad, Fariha Irfan
}

\section{ABSTRACT}

OBJECTIVE: To analyze the quality of intraoral periapical radiographs (IOPA) taken by dental assistants in the department of operative dentistry.

METHODOLOGY: The study design was cross-sectional descriptive type, non-probability convenient sampling technique was used to achieve the calculated sample size. A total of 100 periapical radiographs were collected for evaluation from department of operative dentistry at Hamdard University Dental Hospital, Karachi. Pre, intra and post-operative radiographs were evaluated taken during endodontic therapy. National Radiological Protection Board recommended three point quality scale was used to analyze the IOPA radiographs into excellent, acceptable or unacceptable. The targets were set according to the "Guidance Notes for Dental Practitioners on the Safe Use of X-ray Equipment" recommendations.

RESULTS: A total 100 radiographs were evaluated, $32 \%$ were classified as excellent, $45 \%$ diagnostically acceptable and $23 \%$ of the radiographs diagnostically unacceptable. Rejection rate analysis showed that incorrect film positioning was the most frequent fault resulting in missing or obscured coronal/apical area being the cause in $50 \%$ cases.

CONCLUSION: It has been concluded that frequent quality assessment and monitoring of dental team and their training in dental radiography would help in achieving gold standards. Furthermore, following standard guidelines increase the quality of the radiographs and enhance safety practices.

KEYWORDS: Periapical radiographs, Quality analysis, Dental assistants.

\section{This article may be cited as: Ali SA, Hussain M, Shahzad M, Irfan F. Quality Analysis of Periapical Radiographs taken by Dental Assistants. J Liaquat Uni Med Health Sci. 2018;17(04): 260-4. doi: $10.22442 /$ jlumhs.181740589}

\section{INTRODUCTION}

Radiographs are considered a crucial aid for the diagnosis as well as intra and post-operative evaluation of conditions and different dental procedures ${ }^{1}$. Radiography is the clinician's main diagnostic aid in various disciplines of dentistry ${ }^{2}$. For the diagnosis of majority of intra-oral pathological conditions as well as assisting in endodontic treatment intra-oral periapical radiographs (IOPA) have become an extremely important tool ${ }^{3}$.

During endodontic procedures, periapical radiographs are still the most commonly used method for the localization and presence of periradicular lesions and adjacent anatomical structures ${ }^{1}$. For accurate diagnosis optimal image quality is necessary which the responsibility of professionals performing radiographic examination ${ }^{1-3}$.

Multiple risks are associated with ionizing radiation for radiographic examination and it was recognized soon after its introduction in the field of medicine, therefore methods are introduced to reduce the exposure of ionizing radiation such as protection apparatus, digital radiography, faster speed films etc ${ }^{2}$. Availability of basic knowledge, quality images, and absence of technical and processing errors are necessary factors for correct radiographic interpretation ${ }^{2,3}$, documentation of different types and frequency of errors occurring when radiography performed by professionals and technicians is necessary to identify and correct the existing deficiencies. The number of repetitions and exposures of patient to radiation will thus reduce with the help of such regular clinical audits for dental radiography ${ }^{4,5}$.

Literature review showed that audits on the quality have been frequently reported addressing general issues such as quality of clinical image, processing and record keeping. Such reports impact positively the quality of radiography by increasing the compliance through identification of errors and addressing corrective measures to overcome these $\mathrm{e}^{2-13}$.

A dental assistant provide assistance for treating patient chair-side and is mostly trained on job. In institution based dental hospitals radiographs are usually performed by dental assistants in the dental OPD before, during and after dental treatments especially during operative and endodontic treatments. The aim of this study was to evaluate the quality of 
radiographic images taken by dental assistants.

\section{METHODOLOGY}

The study design was cross-sectional descriptive type, non-probability convenient sampling technique was used to achieve the calculated sample size. Ethical approval was taken from the hospital ethics board. By taking prevalence of inadequate film positioning $(4.4 \%)$, confidence interval $95 \%$, margin of error $5 \%$, the calculated sample size was $n=65$, however the sample size was increased to 100 as consistent with previous study ${ }^{3}$. A total of 100 periapical radiographs were collected for evaluation from department of operative dentistry at Hamdard University Dental Hospital, Karachi from April to May 2017. The radiographs evaluated were taken prior, during and after finishing endodontic therapy. The radiographs were taken by 4 trained male dental assistants having $10,7,3$ and 1 years of experience respectively. An ISO size 2 film (Primax RDX-58E soft) was used to take the radiograph with similar X-Ray units $(60 \mathrm{KV}$, PROX Wireless portable X-Ray unit) and exposure time (0.8 seconds). Processing was done using visual method in acrylic boxes. Film holding device, hemostat and patient's finger were used for positioning the films. All of the radiographs were taken by trained dental assistants having different years of experience. Examination of the films were done by a single examiner to eliminate inter examiner variability who is an expert specialist in the field of operative dentistry. The following parameters were used to analyze each radiograph:

- Cone angulation (Vertical): If the image became long or short, the mistake of cone angulation in vertical direction was detected.

- Cone angulation (Horizontal): If the image of structures in radiograph overlap, the mistake of cone angulation in horizontal direction was detected.

- Positioning of film: Mistake in positioning of film was recognized if the image became Off centered or if crown cut off or root cut off occurred.

- Contrast of image: Mistake in contrast of image was recognized if the image became light or dark.

- $\quad$ Processing of X-ray: Mistake in X-ray processing was recognized if there was improper use of fixative, existence of stains, streaks, and fingerprints.

Quality of radiograph was assessed using National Radiological Protection Board (NRPB) recommendations ${ }^{10}$ as falling into one of the following three categories:

- GRADE 1: Grade 1 radiographs display excellent quality with no faults in positioning or processing. (Excellent)
- GRADE 2: Grade 2 radiographs display some faults in positioning or processing but these faults do not minimize the diagnostic value of radiograph. (Acceptable)

- GRADE 3: Grade 3 radiographs display faults in positioning or processing rendering the radiograph of no diagnostic value. (Unacceptable)

The targets were set according to the 'Guidance Notes for Dental Practitioners on the Safe Use of X-ray Equipment ${ }^{10}$ recommendations as following:

- $70 \%$ or above dental images should have a rating of Excellent.

- $20 \%$ or above dental images should have a rating of diagnostically acceptable.

- $10 \%$ or above dental images should have a rating of Unacceptable.

Data analysis was done using Statistical package for social sciences (IBM SPSS statistics version 22) software using simple descriptive statistics including frequency and percentages.

\section{RESULTS}

Total 100 radiographs evaluated, $32 \%$ scored Grade I, $45 \%$ scored Grade 2 and $23 \%$ of the radiographs scored Grade 3 as shown in figure I. Rejection rate analysis showed that incorrect film positioning was the most frequent fault resulting in missing or obscured coronal/apical area being the cause in 50\% cases followed by incorrect horizontal angulation (20\%), incorrect vertical angulation (15.5\%) and incorrect X-ray processing (12.5\%). $2 \%$ radiographs also shown multiple errors having cone cutting, off centered image and incorrect contrast.

Intra-operative radiographs were found to have more radiographic errors then pre and post-operative endodontic therapy.

Experienced dental assistants were found to made fewer mistakes (10\%) than less experienced dental assistants (70\%). Dental assistant who used film holding devices during development of radiographic image shown least number of errors (5\%), those who used hemostat for holding film made fewer errors $(7.3 \%)$ whereas those who used patient's finger made the most number of errors (45.5\%). Incorrect film positioning was observed as the most frequent radiographic error made by dental assistants with who were less experienced. Table I summarizes the frequency of types of errors made by individual dental assistants according to years of experience.

Female patients were found to have more number of radiographs falling in grade $3(15 \%)$ as compared to males $(8 \%)$ in our study but this observation is not found to be clinically significant ( $p$ value $=0.71$ ). 
Syed Abrar Ali, Mehmood Hussain, Muhammad Shahzad, Fariha Irfan

TABLE I: DISTRIBUTION OF ERRORS ACCORDING TO EXPERIENCE OF DENTAL ASSISTANTS

\begin{tabular}{|l|c|c|c|c|c|}
\hline \multirow{2}{*}{ Type of Error } & \multicolumn{4}{c|}{ Assistant Experience } & \multirow{2}{*}{ Total } \\
\cline { 2 - 5 } & 10 years & 7 years & 3 years & 1 year & \\
\hline None & 08 & 05 & 05 & 09 & 32 \\
\hline Minor error not interrupting diagnosis & 02 & 06 & 05 & 08 & 21 \\
\hline Incorrect vertical angulation of cone & -- & -- & 04 & -- & 04 \\
\hline Incorrect horizontal angulation of cone & -- & 02 & -- & -- & 02 \\
\hline Incorrect film positioning & -- & 01 & 08 & 10 & 14 \\
\hline Light or dark image & 04 & 02 & 04 & 02 & 12 \\
\hline Incorrect x ray processing & 06 & 02 & 03 & -- & 11 \\
\hline Multiple errors & 01 & -- & -- & 03 & 04 \\
\hline Total & 21 & 18 & 29 & 32 & 100 \\
\hline
\end{tabular}

FIGURE I: PERCENTAGE OF RADIOGRAPHS

FALLING IN 3 GRADES

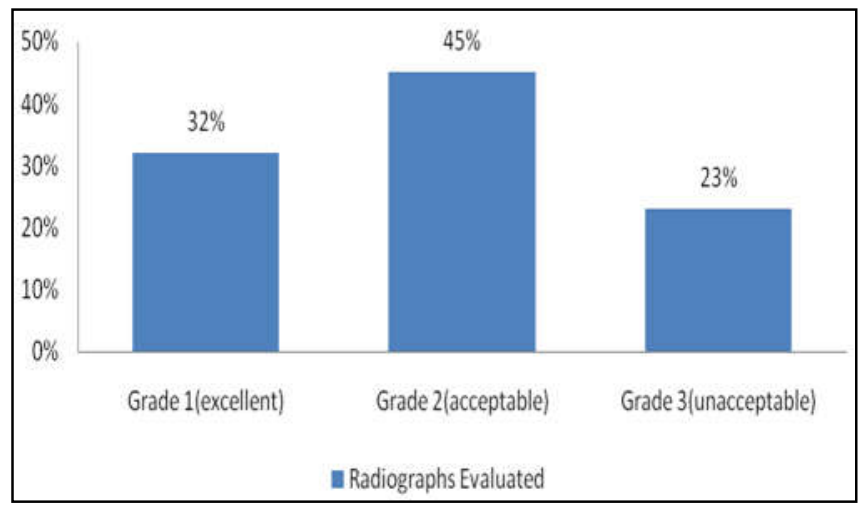

\section{DISCUSSION}

Frequent analysis of radiographs as a part of quality assurance program ensures that the frequency of errors and their causes are continuously monitored ${ }^{4}$. Regular monitoring of quality of radiographs being taken provides a means of simple checking that either members of dental team are meeting or not the expected standards ${ }^{5}$. Addressing these faults provide a mean of improving clinical practice through minimizing the need of repetition of X-rays radiograph thus controlling radiation doses to be As Low As Reasonably Achievable (ALARA) while on the other hand ensures that adequate diagnostic information has been consistently provided along with cost-effectiveness ${ }^{6}$.

Intra-oral periapical radiographs (IOPA) has a crucial role in restorative dentistry as well as in the field of endodontics used for detection of hard and soft tissues pathology pre-operatively, for determination of working length and endodontic mishaps intra-operatively and for the evaluation of obturation and healing post-operatively ${ }^{1,2}$. In our study IOPA radiographs were evaluated for their quality according to the standards provided by NRPB which provides guidance to dental practitioners for radiological protection suggesting that regular audits identify and rectify the common errors improving the quality of radiography.

The result of $32 \%$ radiographs being free of major radiographic errors and demonstrating excellent quality falls short of the recommended target of not less than $70 \%$. Incorrect light contrast of image owing to the improper use of developer/fixer along with presence of streaks and fingerprints is reported in our research as the main error in radiographs which fall in grade 2 .

In our study incorrect film positioning was found to be the most common radiographic error $(50 \%)$ followed by incorrect angulation of cone. This finding is in consistent with Emanuel RJ $2003^{11}$ suggesting that the use of position indicating device (PID) should be made mandatory or at least whenever possible. Ingle JI $2008^{12}$ has reported use of hemostat as film holder as well as cone positioning device. It has been observed in our study that use of hemostat for film positioning performed fewer errors than patient's finger which made most number of errors suggesting that hemostat can be used as an alternative to PID in cases where PID is not available instead of using patient's finger.

Dental assistants were evaluated in our research identifying the areas where they were lacking so that recommendations can be given and implied to improve their performance. It has been observed that those who have greater number of years of experience perform better than the less experienced. 
Film positioning was found to be the most frequent error when performed by the beginner whereas incorrect image contrast appeared to be the most common error with the experienced dental assistants. Therefore less experienced and beginners should be frequently monitored along with training and lectures by professional radiologists.

IOPA Radiographs taken intra-operatively were found to make the maximum number of errors in our research. This can be attributed to the fact that due to rubber dam application film positioning becomes difficult. This problem can be rectified by using extra-oral periapical radiograph. Also in our study female patients were found to have more number of unacceptable radiographs owing to increase gagging reflex in females and fewer thresholds to hold the film intra-orally.

Quality analysis and continuous clinical audits for dental radiography is quite lacking and highly neglected in our region of practice. Local studies has been reported demonstrating the quality assessment of lateral cephalometric radiographs and dental panoramic radiograph by Khan SQ 2017 and $2015^{6,13}$. On best of our knowledge no local researches and documentation are found on literature review on conventional as well as digital IOPA radiography. This indicates that more work needs to be carried out in this domain ensuring maintenance of quality and good clinical practice.

\section{CONCLUSION}

Mastery of evaluation and assessment of dental radiographs is a necessary tool for successful treatment in each and every aspect of dentistry. Along with this it is also crucial that each and every member of dental team must be aware of the causes of errors and faults occurring during radiography and how to address them. Frequent quality assessment of radiographs should be done so as to increase the awareness. Similarly periodic quality assessment of IOPA radiographs can improve clinical practice through radiation dose reduction as well as means of cost-effectiveness.

It has been concluded that frequent quality assessment and monitoring of dental team and their training in dental radiography would help in achieving gold standards. Furthermore, following standard guidelines increase the quality of the radiographs and enhance safety practices.

\section{RECOMMENDATIONS}

On the basis of our results, following recommendations are proposed:

1. To introduce dental Radiology as a separate subject in dental curriculum

2. To include trained dental radiologist in the dental team and there should be a clinical audit at a 6 month interval.

3. Radiographs falling under the category of unacceptable should be documented and analyzed so as to increase awareness about quality issues.

4. Further research is recommended for quality improvement.

\section{REFRENCES}

1. Shah N, Bansal N, Logani A. Recent advances in imaging technologies in dentistry. World $\mathrm{J}$ Radiol. 2014; 6(10):794-807.

2. Felippe MCS, Nassri MRG, Burgos PG, De Freitas SFT, Lage-Marques JL. Quality of periapical radiographs taken by undergraduate students during endodontic treatment. Rev Sul-Bras Odontol. 2009; 6(1):64-9.

3. Almogbel AA, Abdullah A. Quality of periapical radiographs taken by undergraduate dental students at Qassim university. Dent Student Res. 2014; 12(1):10-15.

4. Toy A, Eaton KA. X-Ray Vision: How audit can help you reveal the quality of your radiography. Prim Dent J. 2013; 2(1): 32-9.

5. Salami A, Al Halabi M, Hussein I, Kowash M. An Audit on the Quality of Intra-Oral Digital Radiographs Taken in a Postgraduate Paediatric Dentistry Setting. Oral Health Dent Manag. 2017; 16(1):1-4.

6. Khan SQ, Sobia AH, Mehdi H, Ashraf B. Quality assessment of lateral cephalometric radiographs. Pak Oral Dent J. 2017; 37(2):265-8.

7. Kaviani $F$, Johari M, Esmaeili F. Evaluation of common errors of panoramic radiographs in Tabriz Faculty of Dentistry. J Dent Res Dent Clin Dent Prospects. 2008; 2(3):99-101.

8. Elangovan S, Mahabob MN, Jaishankar S, Kumar BS, Rajendran D. Faulty Radiographs: A cross-sectional analysis among dental college students in Namakkal District, Tamil Nadu, India. J Pharm Bioallied Sci. 2016; 8(Suppl 1): S116S118.

9. Royal College of Radiologists (United Kingdom). Guidelines on radiology standards for primary dental care. Documents of the NRPB. 1994; 5 (3):1-57.

10. NRPB. Guidance Notes For Dental Practitioners On The Safe Use Of X-Ray Equipment. Chilton: National Radiological Protection Board, 2001.

11. Emanuel RJ. A retrospective audit on the quality of periapical and bitewing radiographs taken in a 
primary care setting. Quality in Primary Care. 2003; 11: 305-8.

12. Ingle JI, Bakland LK, Baumgartner JC. Ingle's. Endodontics 6. 6th ed. Bindner $P$, editor. Hamilton, Ontario: BC Decker; 2008.
13. Khan $S Q$, Ashraf $B Q$, Mehdi $H$. Evaluation of Patient Preparation and Positioning Errors on Digital Panoramic Radiographs. Pak Oral Dent J. 2015; 35(1):65-9.

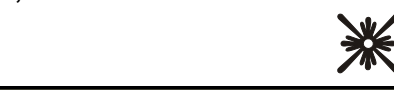

AUTHOR AFFILIATION:

Dr. Syed Abrar Ali (Corresponding Author) Associate Professor, Department of Operative Hamdard University Dental Hospital Hamdard College of Medicine and Dentistry Hamdard University, Karachi, Sindh-Pakistan. Email: dentist4@hotmail.com

Dr. Mehmood Hussain

Associate Professor, Department of Prosthodontic Hamdard University Dental Hospital Hamdard College of Medicine and Dentistry Hamdard University, Karachi, Sindh-Pakistan.

\section{Dr. Muhammad Shahzad}

Associate Professor, Oral and Maxillofacial Surgery Liaquat University of Medical \& Health Sciences

Jamshoro, Sindh-Pakistan.

\section{Dr. Fariha Irfan}

FCPS II Resident, Department of Operative Hamdard University Dental Hospital Hamdard College of Medicine and Dentistry Hamdard University, Karachi, Sindh-Pakistan. 